\title{
A cooperative interaction between U2AF65 and mBBP/SF1 facilitates branchpoint region recognition
}

\author{
J. Andrew Berglund, ${ }^{1}$ Nadja Abovich, and Michael Rosbash ${ }^{2}$ \\ Howard Hughes Medical Institute and Departments of Biology and Biochemistry, Brandeis University, \\ Wal tham, Massachusetts 02254 USA
}

\begin{abstract}
During the early events of pre-mRNA splicing, intronic cis-acting sequences are recognized and interact through a network of RNA-RNA, RNA-protein, and protein-protein contacts. Recently, we identified a branchpoint sequence binding protein in yeast (BBP). The mammalian ortholog (mBBP/SF1) also binds specifically to branchpoint sequences and interacts with the well studied mammalian splicing factor U2AF65, which binds to the adjacent polypyrimidine (PY) tract. In this paper we demonstrate that the mBBP/ SF1-U2AF65 interaction promotes cooperative binding to a branchpoint sequence-polypyrimidine tract-containing RNA, and we suggest that this cooperative RNA binding contributes to initial recognition of the branchpoint sequence (BPS) during pre-mRNA splicing. We also demonstrate the essential nature of the third RBD of U2AF65 for the interaction between the two proteins, both in the presence and absence of RNA.
\end{abstract}

[Key Words: BPS; PYtract; protein-protein interaction; RNA]

Received October 27, 1997; revised version accepted January 26, 1998.

Pre-mRN A splicing is performed by the spliceosome, a large complex consisting of several small nuclear ribonucleoproteins and many non-snRN P proteins. Spliceosome assembly proceeds through several intermediate complexes, beginning with the $\mathrm{E}$ complex, or commitment complex, proceeding to A, B, and, finally, to the C complex, within which chemistry (phosphodiester bond cleavage and formation) takes place. Mammalian splicing substrates contain an intronic 5' splice site, branchpoint sequence (BPS), polypyrimidine (PY) tract, and 3' splice site. The $2^{\prime}-\mathrm{OH}$ of the branchpoint adenosine within the BPS is the nucleophile for attack of the $5^{\prime}$ splice site, which forms a lariat intermediate and a free $5^{\prime}$ exon; in the second step, the $3^{\prime} \mathrm{OH}$ of the liberated $5^{\prime}$ exon attacks the $3^{\prime}$ splice site, resulting in the mRN A and lariat intron products (for review, see Moore et al. 1993; M adhani and Guthrie 1994; Kramer 1996).

During the initial steps of in vitro splicing, the $5^{\prime}$ and $3^{\prime}$ ends of introns are recognized by the U 1 snRN $P$ and other splicing factors, respectively. This corresponds to formation of the commitment complex in yeast and the E complex in mammals (Moore et al. 1993). There are modest sequence requirement differences between the two systems, especially on the 3' side of the intron (BPS, PY tract, $3^{\prime}$ splice site). In yeast, the BPS is the almost

\footnotetext{
${ }^{1}$ Present address: Department of Chemistry and Biochemistry, University of Colorado, Boulder, Colorado 80309 USA.

${ }^{2}$ Corresponding author.

E-MAIL rosbash@binah.cc.brandeis.edu; FAX (781) 736-3164.
}

invariant UACUAAC (Rymond and Rosbash 1992), whereas in mammals, the BPS is degenerate with a consensus sequence of YNCURAY (Keller and N oon 1984; Green 1986). In mammals, a PY tract is highly conserved and an essential 3' side element (Reed and Maniatis 1985; Ruskin and Green 1985). This is much less conserved and important in yeast introns, al though a uri dine tract will modestly enhance yeast splicing (Patterson and Guthrie 1991). The splicing components involved in E complex or commitment complex formation include: (1) U 1 snRN P base pairing to the 5' splice site in both systems (Mount et al. 1983; Zhuang and Weiner 1986; Séraphin et al. 1988; Siliciano and Guthrie 1988); (2) U 2AF65 binding the PY tract (mammalian) and M ud2p, which cross-links to the $3^{\prime}$ si de of yeast introns (Zamore et al. 1992; Abovich et al. 1994; data not shown); (3) SR proteins, which promote U 1 snRN P and U2AF65 binding in $\mathrm{E}$ complex formation in mammals (Kohtz et al. 1994; Staknis and Reed 1994); (4) the recently identified yeast and mammalian branchpoint sequence binding proteins (BBP and mBBP/SF1, respectively), which recognize the BPS (A bovich and Rosbash 1997; Bergl und et al. 1997). mBBP is also known as splicing factor one (SF1), which was initially identified as an essential factor for A complex formation (Kramer 1992; Arning et al. 1996). In both yeast and mammals, a network of interactions between $U 1$ snRN $P$ at the $5^{\prime}$ side and proteins at the $3^{\prime}$ side bridges the two ends of the intron and facilitate intron definition and removal (Reed 1996; Abovich and Rosbash 1997). 


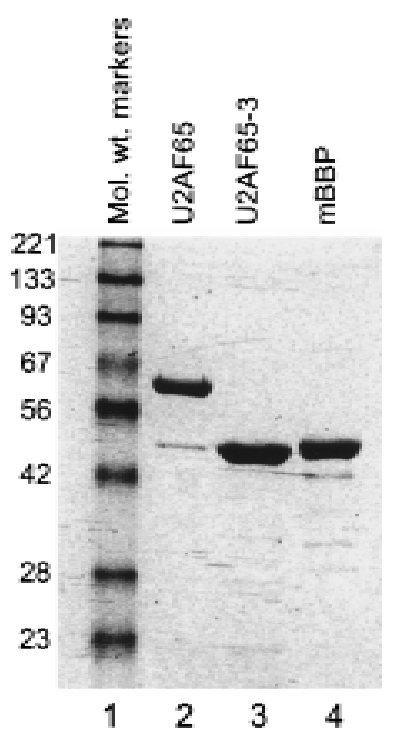

Figure 1. Commassie stained SDS-polyacrylamide gel of purified U2AF65, U2AF65-3, and mBBP. Three micrograms of protein was loaded in each lane. Protein markers are from GIBCO.

The organization of mammalian intron $3^{\prime}$ side sequence el ements (from $5^{\prime}$ to $3^{\prime}$ ) is BPS-PY tract-3' splice site (AG), and the distance from the branchpoint adenosine to the $3^{\prime}$ splice site is normally between 20 and 40 nucleotides (Reed 1989; Smith and N adal-Ginard 1989). Thus, the BPS and PY tract are usual ly adjacent. Because we had shown previously that U2AF65 and mBBP can interact (A bovich and Rosbash 1997), we suspected that this might contribute to intron recognition. A cooperative binding interaction would facilitate BPS selection despite low sequence information content within the degenerate mammalian BPS. Although a similar cooperative interaction may occur between BBP and Mud2p in yeast, this is more difficult to test; the RNA binding properties of Mud2p are not known, and recombinant Mud2p is not available.

For the mammalian proteins, the binding affinity of U2AF65 for the PY tract has been determined, and all three RNA-binding domains (RBDs) affect the strength of the interaction between U2AF65 and the PY tract (Zamore et al. 1992). U2AF65 also has an amino-terminal RS domain, rich in argi nines and serines. RS domains are generally involved in protein-protein interactions within the spliceosome, mediated in part through serine phosphorylation (Fu 1995). However, the RS domain of U2AF65 is unusual, as it interacts with RNA and may not mediate protein-protein interactions: U2AF65-RS has been cross-linked to the BPS and facilitates the annealing of the snRNA in U2 to the BPS in biochemical experiments (Valcárcel et al. 1996).

We have shown more recently that the other mammalian $3^{\prime}$ side factor, mBBP, specifically recognizes the yeast BPS (UACUAAC), which is also the preferred BPS in mammals (Zhuang et al. 1989). The putative RNA binding region of mBBP contains a $\mathrm{KH}$ and a $\mathrm{Zn}$ knuckle domain and is sufficient for specific RN A binding (Berglund et al. 1997). Although mBBP recognizes the BPS, its affinity and specificity are less impressive than those of its yeast ortholog BBP, which recognizes all seven positions within the yeast BPS. In contrast, mBBP binding is only affected by point mutations at the branchpoint adenosine and the conserved uridine two nucl eotides upstream; these are the two most conserved nucleotides in the mammalian branchpoint consensus sequence, $\mathrm{YN}$ CURAY (Keller and N oon 1984). This poorly conserved, short sequence is probably insufficient to specify a mBBP-BPS interaction. We speculated that if U2AF65 were bound to the PY tract, protein-protein contacts with mBBP would facilitate recognition of the BPS (Berglund et al. 1997).

Here, we use recombinant mBBP and U2AF65 to demonstrate a cooperative interaction during binding to a

A

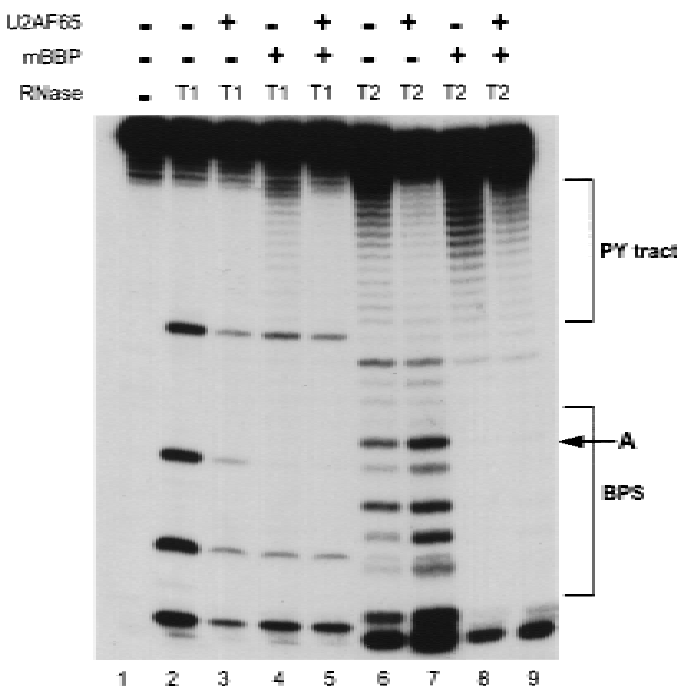

$\mathbf{B}$
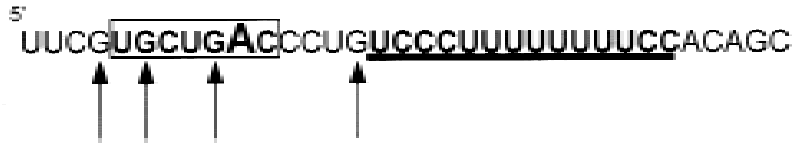

Figure 2. Double footprint of $\mathrm{mBBP}$ and U 2AF65. (A) Purified mBBP, U 2AF65, or both proteins together were incubated with 5 '-end labeled RN A substrate in the presence of either RN ase T1 (lanes 2-5) or RN ase T2 (lanes 6-9), and then run in a denaturing polyacrylamide gel as described in Materials and methods. (Lanes 4,8) The concentration of mBBP used was $11 \mu \mathrm{m}$; (lanes 3,7) concentration of U2AF65 was $2.8 \mu \mathrm{M}$; (lanes 5,9) mBBP was at a concentration of $5.5 \mu \mathrm{M}$ and U 2AF65 at $1.4 \mu \mathrm{m}$. The PY tract and BPS are marked by brackets, and the position of the branchpoint adenosine is marked by an arrow. (B) Sequence of the 34-nucleotide RNA substrate derived from the Adenovirus major late pre-mRN A substrate. The BPS is in bold and boxed, and the branchpoint adenosine is in large text. The PY tract is in bold and underlined. Arrows represent guanosines cleaved by RN ase T1 (lane 2). 
RNA substrate containing a BPS and PY tract. The formation of this ternary complex (mBBP, U2AF65, and RN A substrate) appears to beaided by the presence of the branchpoint adenosine within the BPS as well as by a contiguous PY tract. We also demonstrate the importance of the third RBD of U2AF65, for the physical interaction between U2AF65 and mBBP both in the presence and absence of RNA. The results suggest that cooperative RN A binding by these two proteins constitutes initial recognition of the BPS region and may even contribute to branchpoint selection.

\section{Results}

Cooperative interaction between mBBP and U 2AF65

The nucleotides important for specific recognition by mBBP and U2AF65 have been identified within their respective RN A elements (Zamore et al. 1992; Singh et al. 1995; Berglund et al. 1997). However, footprinting of U2AF65 to RNA has only been assayed with CMCT, which does not examine protection of adenosine, cytosine, or guanosine (Singh et al. 1995). Additional experiments might determine whether either protein interacts with RNA on either side of its recognition site. This might influence cooperative binding by the two proteins. To this end, footprinting experiments were performed with purified full-length recombinant U2AF65, mBBP containing amino acids 1-361 (Fig. 1), and a 34-nucleotide RN A substrate derived from the 3 ' side of adenovirus major late pre-mRNA. In this commonly employed pre-mRNA substrate, the BPS and PY tracts are separated by only four nucleotides (Fig. 2B). If either protein extends past its specific site, this might influence the interaction between the two proteins.

U 2AF65 protects the PY tract but not the BPS (Fig. 2A, Iane 7), and $\mathrm{mBBP}$ protects only the BPS and not the PY tract (Fig. 2A, lane 8). The two proteins, therefore, sit side-by-side, with no more than a 1- or 2-nucleotide space in between. The protection pattern for $\mathrm{mBBP}$ is reminiscent of that observed for the yeast orthol og (BBP); both proteins protect the 7 nucleotides of the BPS and only 2 nucleotides on either side (Bergl und et al. 1997). With U2AF65 as well as mBBP, the protection spans

A

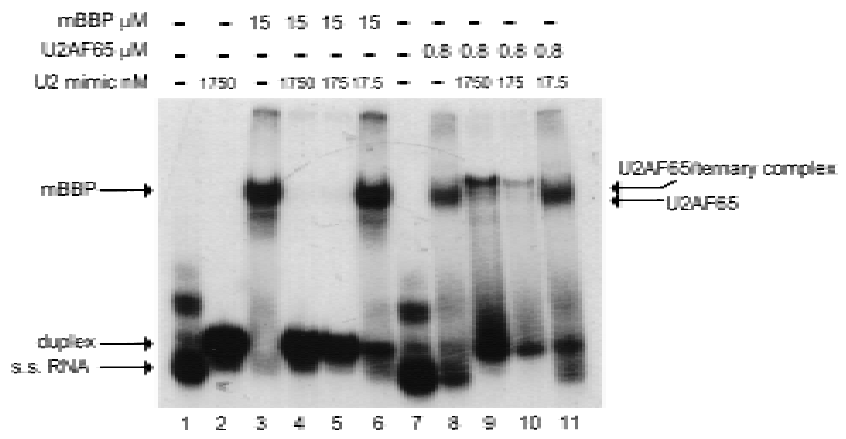

both the BPS and PY tract (Fig. 2A, lane 9). In this case, the protection of the PY tract is weaker (lane 9), probably the result of a low level of contaminating $R N$ ases in the mBBP preparation. Evidence supporting this interpretation is in lane 4: RN ase T1 should have only guanosine cleavages, but addition of $\mathrm{mBBP}$ induces some cleavage of the PY tract.

RNase T1 was assayed primarily for mapping purposes, because the 34 nucleotide RNA contains only five guanosines, two of which are in the BPS (Fig. 2B). The four visible guanosines are all partially protected by U2AF65, possibly because of steric effects on RN ase T 1 activity or to additional U2AF65 molecules that bind nonspecifically to the BPS (Fig. 2B, lane 3). MBBP also protects the four guanosines; protection of three is similar to U2AF65, but mBBP completely protects the guanosine next to the branchpoint adenosine (Fig. 2A, Ianes $4,5)$. This suggests that the complete protection of this one guanosine is the result of a strong mBBP interaction at this position, and the partial protection of the other guanosines is caused by weaker interactions or to steric inhibition of RN ase T1. The complete protection of this particular guanosine recalls the fact that the branchpoint adenosine and adjacent uridine, the nucleotides to either side of this guanosine, have the strongest effect on mBBP binding (Berglund et al. 1997).

To verify the $\mathrm{mBBP}$ footprinting and the sequence-specific interactions of mBBP at the BPS, we used a competition assay involving a 12-nucleotide RNA that basepairs to the BPS as well as to a few nucleoti des upstream and downstream of the BPS. The oligo was designed to encourage a bulged branchpoint adenosine, as has been shown for the U2 snRNP-BPS interaction (Query et al. 1994). This antisense molecule is, therefore, a simplified mimic of U2 snRN P (Fig. 3B, U2 mimic).

Base-pairing of the $U 2$ mimic to the BPS blocks mBBP binding (Fig. 3A, Ianes 4,5). Only at a low concentration of U2 mimic is mBBP binding detectable (lane 6). This suggests that $\mathrm{mBBP}$ and $\mathrm{U} 2$ snRN $\mathrm{P}$ binding are mutually exclusive. In contrast, U2 mimic base-pairing has only a modest effect on U 2AF65 binding, and slightly decreases the mobility of the complex (Fig. 3A, lanes 9,10). The data indicate that U2AF65 can form a ternary complex with the 34-mer and U2 mimic (Valcárcel et al. 1996).

B U2 mimic

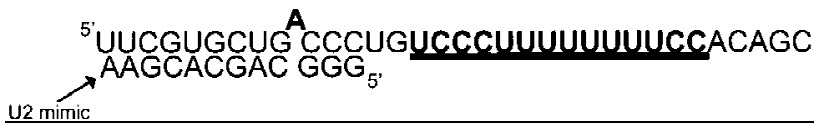

Figure 3. The binding of a 12-nucl eotide RN A to the BPS competes with $\mathrm{mBBP}$ binding. (A) (Lane 1) radiolabeled RN A al one; (lane 2-11) either the 12-nucleotide RNA (U2 mimic), mBBP, U2AF65, or a combination as shown above the autoradiograph. The different complexes are labeled by arrows (right). (B) Sequences of the two RN A oligoribonucleotides. The branchpoint adenosine is shown in bold and bulged out, and the PY tract is in bold and underlined. 
Because mBBP is present in E complex (A bovich and Rosbash 1997), mBBP probably binds to the BPS and is then replaced by U 2 snRNP during or prior to A complex formation.

We used the same RN A substrate (Fig. 2B) and purified proteins to establish assays using native polyacrylamide gel el ectrophoresis. Purified mBBP, U2AF65, or both together were incubated in the presence of radiolabeled RNA, and complexes were then separated on a $6 \% 0.5 \times$ TBE gel (Materials and Methods). The different com- pl exes are easily distinguished (Fig. 4A): Iane 7, U2AF65RNA; lane 5, mBBP-RNA; lane 10, mBBP-U2AF ${ }_{65}^{-}$ RNA. The $K_{D}$ s are $1 \mu \mathrm{M}$ for U2AF65 and $6 \mu \mathrm{M}$ for mBBP. For both binary complexes, binding appears weakly cooperative. This is apparent in previous studies with U2AF65 (Zamore et al. 1992; Lee et al. 1993) and has been generally more discussed for proteins with multiple RNA binding domains (Birney et al. 1993).

The ternary complex of U2AF65, mBBP, and RNA is clearly distinguishable from the binary complexes. Co-
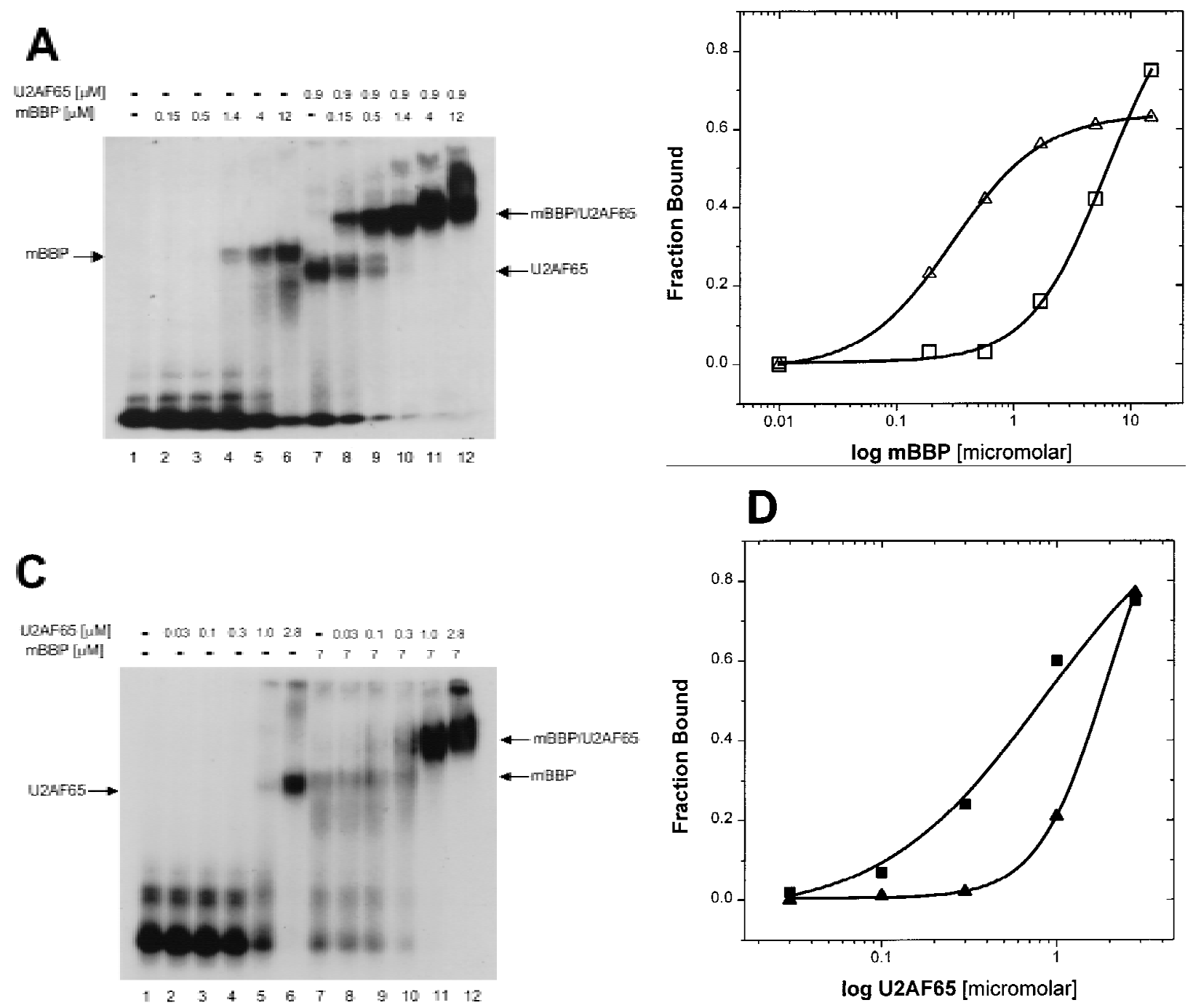

Figure 4. Cooperative binding of mBBP and U2AF65. (A) In a gel-shift assay using a radiolabed 34-nucleotide RN A substrate derived from the Adenovirus major late pre-mRN A and purified proteins MBBP and U2AF65, we assayed for cooperative binding between mBBP and U 2AF65 (M aterials and M ethods). (Lanes 2-6) Increasing concentrations (top) of mBBP; the complex is marked (left) by an arrow. (Lane 7) U2AF65 plus RN A; the complex is again marked by an arrow (right). (Lanes 8-12) U 2AF65 at the same concentration as lane 7 plus mBBP at increasing concentrations, the same as those in lanes 2-6. The ternary complex of mBBP/U2AF65/RNA is marked by an arrow (right). (B) Graphical representation of the data shown in A ( $\square$ ) mBBP; $(\triangle) \mathrm{mBBP}+\mathrm{U} 2 \mathrm{AF} 65$. (C) The same experiment as in A except that U2AF65 concentration is varied (at top); the concentration of mBBP is held constant at $9 \mu \mathrm{M}$. The

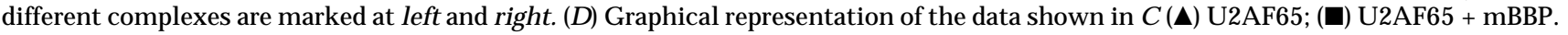
These experiments were repeated multiple times under multiple conditions with approximately the same 20 -fold and 5 -fold effects in cooperativity observed. 
operativity between U2AF65 and mBBP was defined as the ability of one protein to increase the affinity of the other for RN A. A comparison of lanes 2-6 (mBBP) with lanes 8-12 (mBBP plus U2AF65) indicates cooperative RNA binding by the two proteins. In the presence of U2AF65, mBBP forms a complex at $0.57 \mu \mathrm{M}$ (lane 9) but fails to bind at this same concentration without U2AF65 (lane 3). Quantification reveals a 20-fold increase in mBBP's RN A affinity by U2AF65 (Fig. 4B). The effect is based on the difference in apparent $K_{d} S$ (Materials and M ethods): $6 \mu \mathrm{m}$ in the presence of RN A al one and $0.3 \mu \mathrm{m}$ with U 2AF65. The same effect was observed at different U2AF65 concentrations (see legend to Fig. 4; data not shown), but the presence of multiple complexes at saturating U2AF65 concentrations made identification and interpretation of the various complexes difficult.

The experiment was also performed in the opposite way, namely, mBBP concentration was held constant and U2AF65 concentration was varied. With this protocol, cooperativity was al so observed, but the effect was less strong: 5-fold compared with 20-fold (Fig. 4C,D). On the basis of thermodynamics, cooperativity should be independent of which protein concentration is held constant and which is varied. The difference could be the result of a failure to achieve equilibrium under the experimental conditions used. For all cooperative binding assays, incubations were for $1 \mathrm{hr}$ at room temperature before separation by native gel electrophoresis. In any case, both protocols indicate that mBBP and U2AF65 bind in a cooperative manner to an RNA substrate containing a BPS and PY tract.

The effect of RNA mutations on the cooperative interaction between U 2AF65 and MBBP

To determine the contribution of the substrate RNA elements to the cooperative interaction, we assayed mutations within the BPS and PY tract. A mutation changing the branchpoint adenosine to a cytidine was made (indicated by an * above the change); this decreased the mBBP binding affi nity and al so changed the complex mobility (cf. Figs. 5A, lanes 5 and 6 , and 4A). The decreased affinity agrees with our previous work demonstrating that a mutant branchpoint adenosine within the context of a yeast BPS (UACUAAC) decreased mBBP binding (Berglund et al. 1997). The mobility change could be caused by the binding of multiple mBBP molecules or to mBBP binding to a different region of the RN A substrate. In the presence of U2AF65 (Fig. 5A, lanes 8-12), mBBP may still bind weakly to the mutant BPS, on the basis of a similar migration of the ternary complex (Fig. 5A, Ianes 10-12, indicated by an arrow) to the mobility of the ternary complex with wild-type RN A (Fig. 4A). The amount of ternary complex is greatly reduced by mutating the branchpoint adenosine. Because of the weak and aberrant binding of $\mathrm{mBBP}$, it is difficult to determine the extent to which cooperative binding to the mutant substrate is reduced. It is cl ear, however, that U 2AF65 is not able to easily recruit $\mathrm{mBBP}$ in the absence of a consensus BPS.

To determine the effect of mutations in the PY tract, we changed two central uridines to guanosines [**; (Fig. $6 \mathrm{~B})$ ]. In this experiment, mBBP concentration was constant and the concentration of U 2AF65 was varied (Fig. $6 A$ ). A s expected, the PY tract mutation reduces U2AF65 binding (lanes 2-6) compared with the wild-type RNA substrate (Fig. 4C). With this substrate, however, U2AF65 binding is still enhanced in the presence of mBBP (Fig. 6A). This is based on the appearance of the ternary complex at a lower concentration of U2AF65 compared with U2AF65 binding without mBBP (Fig. 6A, cf. lanes 5 and 11). Under these conditions (constant mBBP and variable U2A F65), the cooperative binding interaction to the wild-type RNA is fivefold, and these mutations within the PY tract reduce it (cf. Figs. 4C and $6 \mathrm{~A}$ ). Thus, mutations in both the BPS or PY tract reduce formation of a proper ternary complex.

Domains within U 2AF65 and mBBP important for cooperativity

All four domains of U2AF65 have been shown to be involved in RNA interactions (Fig 7A; schematic representation): All three RBDs contribute to binding with a long

\section{A}

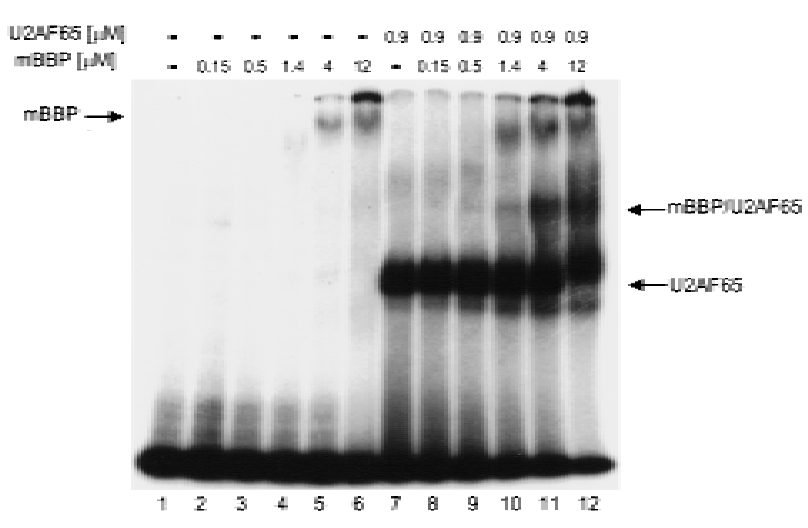

B U. UCGUGCUGCCCCUguCcCUUUUUUUUCCACAGC

Figure 5. Mutation of the branchpoint adenosine reduces formation of the ternary complex (mBBP, U2AF65, and RNA substrate). (A) A 34-nucleotide RN A substrate with the branchpoint adenosine mutated gel-shift experiments was used to perform under the same conditions as those in Fig. 4A. The mBBP/RN A complex has a different migration pattern as marked by an arrow to the side of the autoradiograph. (B) Sequence of the mutated 34-nucleotide RNA substrate used in this experiment. The branchpoint adenosine was changed to cytidine and is marked by an asterisk (*). 


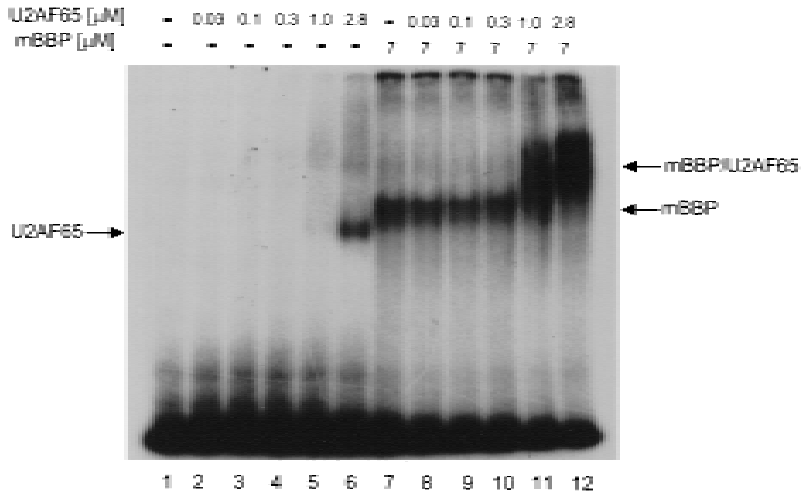

PY tract, and the RS domain cross-links to the BPS and acts as a chaperone for $\mathrm{U} 2$ binding to the BPS (Zamore et al. 1992; Gaur et al. 1995; Val cárcel et al. 1996). To determine which domain(s) contribute to the mBBP interaction, we used yeast two-hybrid and GST-precipitation assays.

Figure 7A summarizes the yeast two-hybrid results. All clones containing the complete third RBD manifested an interaction with $\mathrm{mBBP}$ similar to that observed with full-length U2AF65. Only clone $P$ (Fig. 7A) is inconsistent with this conclusion. Clone $\mathrm{P}$ is a false-positive, because in the absence of $\mathrm{mBBP}$ (glucose is added to repress $\mathrm{mBBP}$ expression) the same strong activation is seen (data not shown). To confirm the two-hybrid re-
B

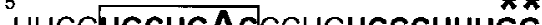

Figure 6. Mutating the $P Y$ tract reduces cooperative formation of the ternary complex (mBBP, U 2AF65, and RNA substrate). (A) This is the same experiment as in Fig. $4 \mathrm{C}$ except the PY tract within the 34-nucleotide RN A substrate has been mutated. The different complexes are indicated to left and right. (B) Sequence showing the double mutation of the PY tract. Two uridines in the middle were changed to guanosine, as indicated by asterisks $(* *)$.

sults, we made yeast extracts containing several of the different fusion proteins and used GST-mBBP to precipitate these LeXA-U2AF65 chimeric proteins. Full-length U 2AF65, clones containing the third RBD and the third RBD alone, interacted with GST-mBBP (Fig. 7B, lanes $10,12,14)$. Clone $\mathrm{P}$ (lane 16) was not recovered, consistent with the false-positive interpretation. The two-hybrid and precipitation results indicate that the third RBD of U2AF65 is the relevant domain for interaction with mBBP.

U2AF65 missing the third RBD (U2AF65-3) was then expressed, purified (Fig. 1; Materials and Methods) and used in the gel-shift assay to determine the rel evance of the third RBD to the cooperative RN A-binding interac-

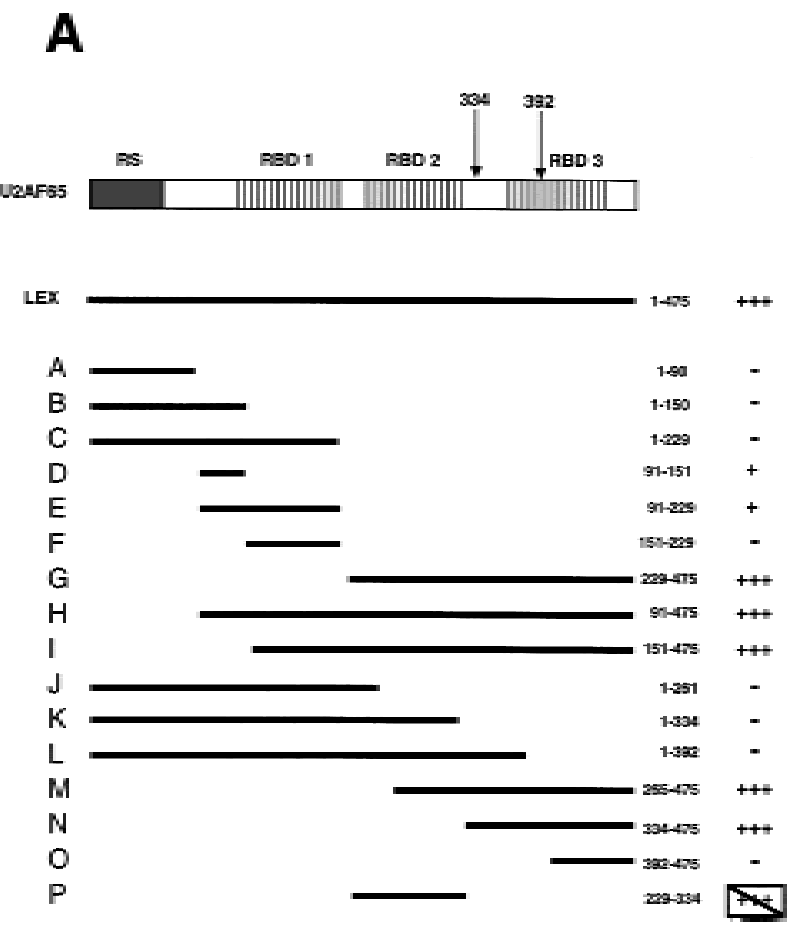

B
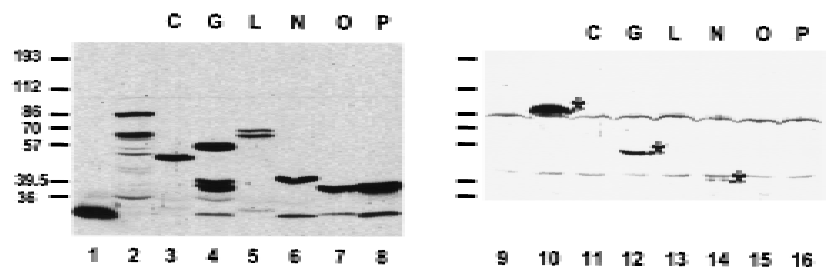

Figure 7. $m B B P$ interacts with the carboxy-terminal RBD of U2AF65. (A) Y east two-hybrid interactions. Y east cells carrying the B42-mBBP fusion and the indicated LexA-U2AF65 fusions were obtained and tested for B-gal actosi dase production as described in $\mathrm{Ma}$ terials and M ethods. A schematic representation of U2AF65 depicting the amino-terminal RS domain and the three RNA-binding domains RBD 1-3. The bars indicate the U 2AF65 region present in each fusion and the numbers correspond to the U2AF65 amino acids fused to LexA. Plus $(+)$ and minus $(\rightarrow$ signs indicate $\beta$-gal actosi dase activity. The box around the three pluses of the $P$ clone indicates that this fusion activates transcription independently of B42-mBBP expression. (B) LexA-U2AF65 fusions interacting with GST-mBBP. (Lane 1) Whole cell extracts were prepared from yeast cells carrying LexA al one, (lane 2) LexA-U2AF65, or (lanes 3-8) the indicated LexAU2AF65 fusions, and $15 \mu \mathrm{l}$ was incubated with the GST-mBBP fusion protein bound to glutathione agarose beads as described in $\mathrm{Ma}$ terials and Methods. The bound proteins were eluted in SDS sample buffer and separated in 8\% polyacrylamide-SDS gels. After transfer to nitrocellulose the LexA fusion proteins were visualized with anti-LexA antibody. (Left panel) corresponds to $10 \mu l$ of the extracts directly loaded on the gel, and (right) proteins after elution from the GST-mBBP beads. 
tion of U2AF65 and mBBP. As previously reported (Zamore et al. 1992), U2AF65-3 binds to the 34 nucleotide RNA with a similar affinity to that of full-length U2AF65. This presumably reflects the rather short nature of the PY tract of this substrate. In contrast to U2AF65, however, U2AF65-3 cannot promote cooperative binding of mBBP to RNA (Fig. 8). A ternary complex is still formed (lanes 11,12 ) but only at concentrations where mBBP alone is al so able to bind (cf. lanes 5,6 and $11,12)$. Therefore, removal of the third RBD of U2AF65 eliminates a detectable physical interaction between U 2AF65 and MBBP, both in the absence and presence of RNA.

We had shown previously that the region of mBBP containing the $\mathrm{KH}$ domain and $\mathrm{Zn}$ knuckle is sufficient for specific binding to the BPS (Berglund et al. 1997). To determine if this same region is sufficient for the cooperative interaction with U2A F65, we used a protein containing amino acids 135-308 from mBBP, termed $\operatorname{mBBP}(181)$, in the cooperativity assay. $\operatorname{mBBP}(181)$ binds 10-fold weaker to this RNA substrate compared with the mBBP (1-361) used previously, suggesting that the amino-terminal portion of mBBP contributes to RNA binding affinity. Importantly, a ternary complex with U 2AF65 is visible, but there is no detectable cooperative interaction between the two proteins (data not shown). Taken together with other data, the result provisionally assigns the relevant interacting region of $\mathrm{mBBP}$ to the amino-terminal end of the protein (see Discussion).

\section{Discussion}

The footprinting data demonstrate that $\mathrm{MBBP}$ and U2AF65 can simultaneously interact with this model RNA substrate in a side-by-side manner (Fig. 2). On the basis of experiments in splicing extracts (Abovich and Rosbash 1997), these interactions probably occur during or prior to $\mathrm{E}$ complex formation. As this step precedes $\mathrm{U} 2$ snRNP addition to the prespliceosome (Michaud and Reed 1991), mBBP binding probably reflects the initial recognition of the BPS.

Previous results indicated that $\mathrm{mBBP}$, as well as BBP, might interact with the bases of the branchpoint region, and the mutually exclusive binding of MBBP or the U2 mimic (Fig. 3) is consistent with this interpretation. This indicates that U 2 snRNP addition and formation of the U2 snRN A-BPS duplex destabilizes mBBP, and there is some indication that this protein may no longer be present in the U2 snRN P-containing A complex (R. Reed, pers. comm.). In contrast, U2AF65 is an A complex component as well as an E complex component. Consistent with this role, U2AF65 can form a ternary complex with the U 2 mimic and the 34 nucleotide RN A substrate (Fig. 3). Formation of this complex is consistent with the previous suggestion that the U 2A F65RS domain contributes to BPS-U2snRNA base-pairing (Valcárcel et al. 1996). The third RBD of U2AF65 interacts with the SAP155 component of U2 snRN P as well as with mBBP (O. Gozani, J. Potashkin, and R. Reed, in prep.). Although it is not known that precisely the same subregion interacts with both proteins, it is tempting to speculate that during this subsequent role of U 2AF65 in U 2 snRN P recruitment a swap of protein-protein interactions also helps displace mBBP from the spliceosome (Fig. 9).

The double footprint (Fig. 2) shows that the two proteins can bind simultaneously to their adjacent sites. Taken together with protein-protein interaction studies (Abovich and Rosbash 1997), the results suggested that RNA binding might be cooperative, which is the case (Fig. 4). It is not yet known whether this is the result of a change in off-rate or on-rate, nor is it known for the proposed effect of U2AF65 on U 2snRN A-BPS annealing (Valcárcel et al. 1996). But even without a proper biophysical explanation, the mBBP-U2AF65 cooperativity suggests features of mammalian BPS selection and more generally sheds light on intron recognition. Cooperative binding increases the specificity of each protein for its respective site and links the BPS and PY tract together as a large recognition site on the $3^{\prime}$ side of mammalian introns. This notion hel ps explain how the highly degenerate mammal ian BPS is recognized by mBBP: Its binding site is both RNA and protein, the BPS and the adjacent U2AF65. Although the PY binding site of U2AF65 may have more sequence information, $\mathrm{mBBP}$ and the BPS

\footnotetext{
Figure 8. The third RBD of U 2AF65 is necessary for the cooperative interaction between $\mathrm{MBBP}$ and U2AF65. Under the same conditions as those for the experiment shown in Fig. 4A (M aterials and M ethods), we looked for a cooperative interaction between $\mathrm{mBBP}$ and a U 2AF65 protein missing the third RBD (U2AF65-3). The three different complexes are marked by arrows at left and right.
}

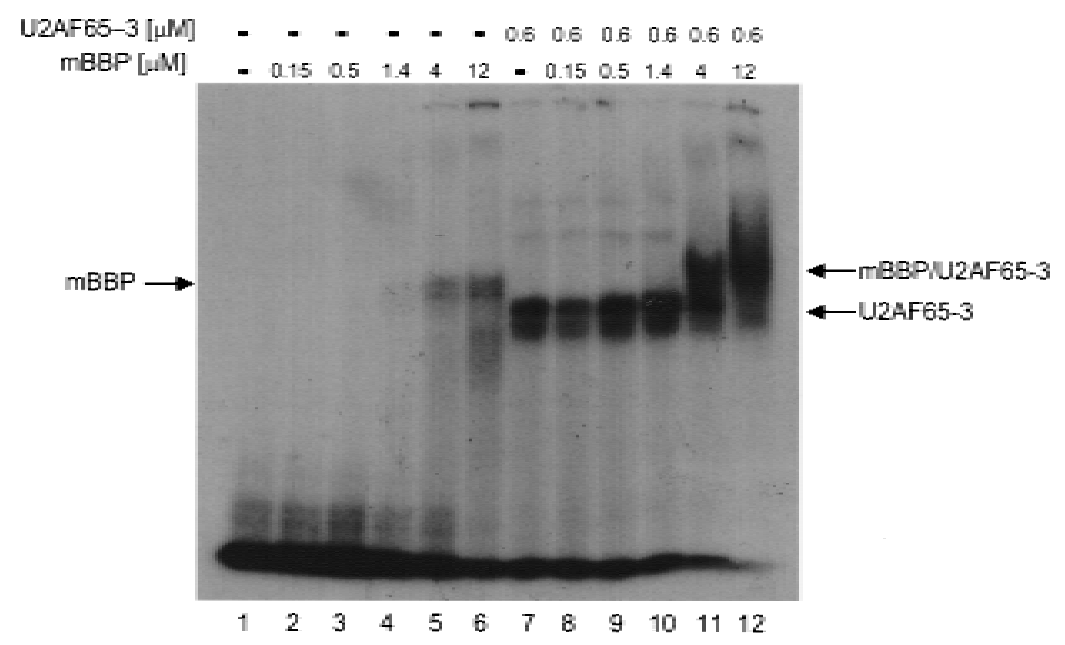




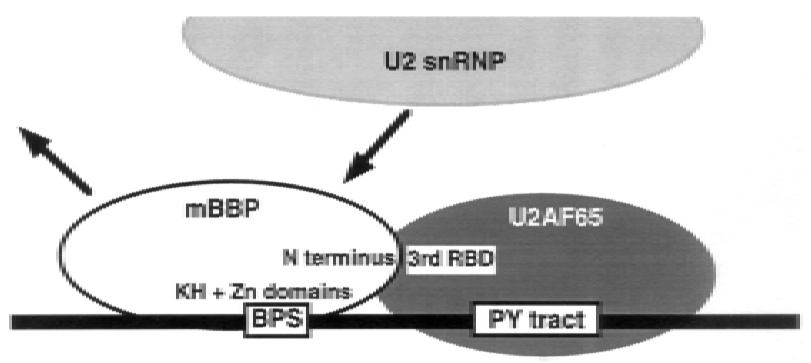

Figure 9. A model representing the cooperative interaction between mBBP and U2AF65 at the $3^{\prime}$ end of mammalian introns and the subsequent replacement of $\mathrm{mBBP}$ by $U 2$ snRN P. The RN A is represented by the thick black line. The important domains within mBBP (amino terminus) and U2AF65 (third RBD) for the cooperative interaction are shown interacting with one another. The $\mathrm{KH}$ domain and $\mathrm{Zn}$ knuckles are shown binding the BPS.

may also be viewed as part of the U2AF65 binding site. As mutations in the RNA substrate have demonstrable effects (Figs. 5 and 6), proper RNA-protein interactions also contribute to the wild-type cooperative interaction. The aberrant mobility of the mBBP-branchpoint mutant complex (Fig. 5) might indicate mispositioning of the protein on this substrate, or it might reflect a missing RNA-induced conformational change necessary for the cooperative protein-protein interaction.

We observed a substantial decrease in the amount of ternary complex (mBBP, U2AF65, and the RNA substrate) formed in the presence of mutations in either the BPS or PY tract (Figs. 5 and 6). Although interpretation of the BPS mutation is less certain, the PY tract mutation has a clear effect on cooperativity. Mutations in either the BPS, PY tract, or the presence of a nonconserved BPS have been shown previously to reduce spli cing efficiency (Hartmuth and Barta 1988; Reed and Maniatis 1988; Reed 1989). Also, formation of the early splicing complex $A$ has been shown to be effected by mutations in either the BPS or PY (Jamison and Garcia-Blanco 1992; Query et al. 1996). Possibly more relevant is the strong effect of branchpoint mutations, particularly the branchpoint adenosine, on complex formation. These studies used partial RNA substrates missing a $5^{\prime}$ exon and a $5^{\prime}$ splice site, or very short substrates similar to the ones used in our studies (Query et al. 1996, 1997). Taken together, all of these results suggest that the decreases we observed in ternary complex formation and cooperativity may correlate with decreases in complex formation and splicing efficiency.

In addition to contributing to branchpoint recognition, the mBBP-U2AF65 interaction may also contribute to formation or stabilization of $E$ complex. Additional interactions with mBBP or U2AF65 have been postulated or are easy to imagine during this subsequent step of spliceosome assembly: (1) interactions between mBBP/ U 2A F65 and U 1 snRN P (A bovich and Rosbash 1997; Fromont-Racine et al. 1997); (2) a cooperative interaction between splicing factors that bind enhancers and the mBBP/U 2AF65 complex (Reed 1996); (3) interactions be- tween U2AF65 and U2AF35 (Zamore and Green 1989); (4) interactions between U2AF35 and U 1 snRNP (Wu and $\mathrm{M}$ aniatis 1993).

The interaction between MBBP and U2AF65 is reminiscent of previously observed cooperativity between $\lambda$ repressor molecules and operator DNA Johnson et al. 1981; Ptashne 1984, 1992). The protein-protein interaction between repressor molecules is relatively weak, only 1-2 kcal/mole Johnson et al. 1979; Ackers et al. 1982). Assuming the cooperativity observed between U2AF65 and mBBP is relevant to the interaction energy between U2AF65 and mBBP, a 20-fold effect is equal to $1.6 \mathrm{kcal} / \mathrm{mol}$, within the same range as that seen for the $\lambda$ repressor. The $K_{D} S$ for $R N A$ binding are greater than those between repressor and DNA, however, indicating that the protein-protein interaction might make a greater relative contribution to the protein-RN A interactions. Alternatively, our in vitro experiments might underestimate the effective affinities of these two proteins for many RN A targets. For example, RN A binding of U2AF65 is improved by lengthening the PY tract (Zamore et al. 1992). Although nothing similar has been achieved for mBBP, additional protein-protein interactions within the $\mathrm{E}$ complex almost certainly make in vivo binding stronger than what is observed in vitro with recombinant proteins. Finally, relatively weak binding of mBBP may be desirable, as it is probably replaced by $U 2$ snRNP during later spliceosomal assembly steps; a strong mBBP-BPS interaction might be rate-limiting and inhibit U2 snRN P addition.

A similar cooperative interaction probably occurs during yeast intron recognition. BBP, the yeast ortholog of mBBP, binds the yeast BPS (Bergl und et al. 1997). Mud2p, the possible yeast ortholog of U2AF65, interacts with BBP (Abovich and Rosbash 1997). Mud2p cross-links to pre-mRN A and may interact with the weakly conserved yeast PY tracts (A bovich et al. 1994). The third RBD of U2AF65 is the region of conservation between U2AF65 and $M u d 2 p$ (Abovich et al. 1994). This is the region of U2AF65 that interacts with mBBP (Fig. 7), and it is al so the region of $M u d 2 p$ that interacts with yeast BBP (J.-C. Rain, Z. Rafi, Z. Rhani, P. Legrain, and A. Kramer, in prep.). Y east BBP and $\mathrm{mBBP}$ al so share a region necessary for the interaction with their respective partners, M ud2 $p$ and U2AF65. This is the amino-terminal portion of BBP, upstream of the KH domain (J.-C. Rain, Z. Rafi, Z. Rhani, P. Legrain, and A. Kramer, in prep.), which is highly conserved between the two proteins (Arning et al . 1996). The conservation al so suggests that this region contributes in a similar manner in both systems to intron recognition.

In most mammalian introns, the BPS and PY tract are close together (Reed 1989). It has been suggested that the proximity of the BPS to the PY tract allows U2AF65 to aid in U2 snRNP addition to the BPS (Zamore et al. 1992), and more recent experiments support this hypothesis (Valcárcel et al. 1996). On the basis of the results reported here, we suggest that this proximity is also important for the earlier cooperative interaction between mBBP and U2AF65. The footprinting experiment (Fig. 2) indicates that a 4-nucleoti de separation does not inhibit 
binding to the two sites. But it will be interesting to determine whether longer or shorter spacing affects the cooperative formation of this ternary complex, because separating the BPS and PY tract have been shown to have a deleterious effect on both $A$ complex formation and lariat formation (Reed 1989; O. Gozani and R. Reed, pers. comm.). A longer PY tract, which has been shown to bind U2AF65 more tightly (Zamore et al. 1992), might even change U2AF65 conformation and thereby affect its interaction with $\mathrm{mBBP}$.

\section{Materials and methods}

\section{Cloning and protein purification}

Plasmids for the production of proteins in Escherichia coli were constructed in the pGEX-6p-1 vector (Pharmacia) by standard PCR amplification with oligonucleotides that introduced re striction sites for cloning: pGEX6P-U2AF65 contains the complete coding region of U2AF65 (amino acids 1-476) flanked by BamHI and EcoRI sites; pGEX6P-U2AF65 $\Delta$ rd RBD contains amino acids 1-364; pGEX6P-mBBP/SF1 contains amino acids 1-361 of mBBP flanked by BamHI and Sall sites.

All three protein contructs (U 2AF65, U 2AF65-3, and mBBP) were transformed into BL21 cells (N ovagen). Cells were resuspended in $50 \mathrm{~mm}$ Tris (pH 7.5), $200 \mathrm{~mm} \mathrm{~N} \mathrm{aCl}, 1 \mathrm{~mm}$ EDTA, and $1 \mathrm{~mm}$ DTT. Cells were sonicated, spun at $17,000 \mathrm{~g}$ for $30 \mathrm{~min}$, and bound to glutathione-Sepharose. Following the protocol from Pharmacia, the protein of interest (U2AF65, etc.) was cleaved from the matrix by use of Precision protease (Pharmacia) and dialyzed overnight against $25 \mathrm{~mm}$ Tris (pH 7.5), $25 \mathrm{~mm}$ $\mathrm{NaCl}, 1 \mathrm{~mm}$ EDTA and $1 \mathrm{~mm}$ DTT. The two U2AF65 proteins were bound to heparin-Sepharose (Pharmacia) and el uted with a salt gradient of $25 \mathrm{~mm}-1 \mathrm{M} \mathrm{N} \mathrm{aCl}$. Peak fractions were collected and concentrated by use of an Amicon ultrafiltration cell and then dialyzed against $25 \mathrm{~mm}$ Tris (pH 7.5), $50 \mathrm{~mm} \mathrm{NaCl}, 1 \mathrm{~mm}$ DTT and $15 \%$ glycerol. Everything was done exactly the same for mBBP except the column matrix used was CM-Sepharose (Pharmacia).

\section{RNA substrates}

All RNA oligonucleotides were made with Perseptive RNA amidites on an Expedite 8909 Oligonucleotide Synthesizer. RNAs were kinased with $\left[\gamma_{-}{ }^{32} \mathrm{P}\right] \mathrm{ATP}$, gel purified, and further purified on a Bio-Rad P6 spin column.

\section{Footprinting assay}

Either mBBP, U2AF65, or both were incubated with radiolabeled RN A in binding buffer [25 mM Tris (pH 7.5), $25 \mathrm{~mm} \mathrm{~N} \mathrm{aCl}$, $1 \mathrm{~mm}$ EDTA], $20 \mathrm{mg} / \mathrm{ml}$ tRN A, and RN asin (Promega) at final concentration of $1 \mathrm{U} / \mathrm{ml}$. After 20 min incubation, either RN ase $\mathrm{T} 1$ (Ambion, at a final concentration of $0.4 \mathrm{U} / \mathrm{ml}$ ) or RN ase T2 (GIBCO, at a final concentration of $0.05 \mathrm{U} / \mathrm{ml}$ ) was added. This mixture was incubated at RT for $5 \mathrm{~min}$, and the reaction was then quenched with phenol/chloroform. After ethanol precipitation, products were separated on a $20 \%$ denaturing polyacrylamide gel.

\section{Gel-shift assay}

Proteins were incubated with radiolabeled RNA in binding buffer plus $0.5 \mathrm{mg} / \mathrm{ml}$ tRN A for $60 \mathrm{~min}$ at room temperature.
Separation of the RNA and the different complexes was done in $0.5 \times$ TBE $6 \%$ native polyacrylamide gels. Running time was $\sim 5$ $\mathrm{hr}$ at $100 \mathrm{~V}$ in the cold room. Radiolabeled RNA was at a final concentration of $\sim 0.1 \mathrm{~nm}$. The $\mathrm{K}_{\mathrm{d}}$ of mBBP alone was obtained by plotting fraction bound (mBBP-RNA complex) versus unbound (free RNA) by use of the program Microcal Origin (Microcal Software Inc.). In the case of the ternary complex, fraction bound was the ternary complex only and unbound was both free RNA and the U2AF65-RNA complex.

The competition assay in which a U2 mimic (12 oligoribonucleotide as shown in Fig. 3) was added to compete mBBP binding was done in a similar manner as above, except that cold U2 mimic was anneal ed to the radiolabeled 34 oligoribonucleotide at $65^{\circ} \mathrm{C}$ for $5 \mathrm{~min}$ and then placed at room temperature (this was done in binding buffer plus $200 \mathrm{~mm} \mathrm{NaCl}$ ). After $10 \mathrm{~min}$, proteins were added. A $7.5 \% 0.5 \times \mathrm{TBE}$ gel was used instead of a $6 \%$ gel.

\section{Two-hybrid and GST precipitations}

The LexA-U2AF65 deletions A-P were a gift from Or Gozani and Robin Reed (Harvard Medical School, Boston, MA). The B42-mBBP/SF1 fusion has been described previously (A bovich and Rosbash 1997). Y east two-hybrid assays were performed in diploids obtained by mating strain EGY 48-psH 18 transformed with each of the LeXA-U2AF65 fusions to strain RFY 206-psH 18 carrying the B42-mBBP/SF1 fusion. After selection of the diploids in $\mathrm{His}^{-}, \mathrm{Ura}^{-}, \mathrm{Trp}^{-}$selective plates, they were replicaplated to selective indicator plates containing X-gal and either gal actose-raffinose or glucose as carbon source.

GST-mBBP/SF1 precipitation of LexA-U2AF65 fusion proteins was performed as previously described (Abovich and Rosbash 1997), except that yeast miniextracts were prepared as described (A bovich et al. 1990). The LexA-U2AF65 fusions were visualized with anti-LexA antibody, a generous gift from Roger Brent (Harvard Medical School, Boston, MA).

\section{Acknowledgments}

We thank Or Gozani and Robin Reed for the generous gift of the LexA-U2AF65 fusions, and communication of results before publication. We also appreciate Pierre Legrain and Angela Kramer communicating results before publication. We are grateful to Melissa Moore and members of the Rosbash laboratory for hel pful discussions and critical reading of the manuscript. Our thanks also go to Ed Dougherty and Lise-Anne Monaghan for hel $\mathrm{p}$ with figures and secretarial assistance. Supported by $\mathrm{Na}$ tional Institutes of Health grant (GM-23549) to M.R.

The publication costs of this article were defrayed in part by payment of page charges. This article must therefore be hereby marked "advertisement" in accordance with 18 USC section 1734 solely to indicate this fact.

\section{References}

Abovich, N . and M. Rosbash. 1997. Cross-intron bridging interactions in the yeast commitment complex are conserved in mammals. Cell 89: 403-412.

Abovich, N., P. Legrain, and M. Rosbash. 1990. The yeast PRP6 gene encodes a U 4/U 6 small nuclear ribonucleoprotein particle (snRN P) protein, and the PRP9 gene encodes a protein required for U2 snRN P binding. Mol. Cell. Biol. 10: 64176425.

Abovich, N., X.C. Liao, and M. Rosbash. 1994. The yeast MUD2 protein: An interaction with PRP11 defines a bridge between commitment complexes and U 2 snRN P addition. Genes \& 
Dev. 8: 843-854.

Ackers, G.K., M.A. Shea, and A.D. Johnson. 1982. Quantitative model for gene regulation by lamba phage repressor. Proc. Natl. Acad. Sci. 79: 1129-1133.

Arning, S., P. Gruter, G. Bilbe, and A. Kramer. 1996. Mammalian splicing factor SF1 is encoded by variant CDNAs and binds to RNA. RNA 2: 794-810.

Berglund, J.A., K. Chua, N. A bovich, R. Reed, and M. Rosbash. 1997. The splicing factor BBP interacts specifically with the pre-mRN A branchpoint sequence UACUAAC. Cell 89: 781787.

Birney, E., S. Kumar, and A.R. Krainer. 1993. Analysis of the RN A-recognition motif and RSRGG domains: Conservation in metazoan pre-mRN A splicing factors. Nucleic Acids Res. 21: 5803-5816.

Fromont-Racine, M., J.-C. Rain, and P. Legrain. 1997. Toward a functional analysis of the yeast genome through exhaustive two-hybrid screens. Nature Genet. 16: 277-282.

Fu, X.-D. 1995. The superfamily of arginine/serine-rich splicing factors. RNA 1: 663-680.

Gaur, R., J. Valcarcel, and M. Green. 1995. Sequential recognition of the premRNA branch point by $U 2 A F^{65}$ and a novel spliceosome-associated 28-kDa protein. RNA 1: 407-417.

Green, M.R. 1986. PremRNA splicing. Annu. Rev. Genet. 20: 671-708.

Hartmuth, K. and A. Barta. 1988. Unusual branch point selection in processing of human growth hormone pre-mRNA. Mol. Cell. Biol. 8: 2011-2020.

Jamison, S.F. and M.A. Garcia-Blanco. 1992. An ATP independent U2 small nuclear ribonucleoprotein particle/precursor mRNA complex requires both splice sites and the polypyrimidine tract. Proc. Natl. Acad. Sci. 89: 5482-5486.

Johnson, A.D., B.J. Meyer, and M. Ptashne. 1979. Interactions between DNA-bound repressors govern regulation by the lamba phage repressor. Proc. N atl. Acad. Sci. 76: 5061-5065.

Johnson, A.D., A.R. Poteete, G. Lauer, R.T. Sauer, G.K. Ackers, and M. Ptashne. 1981. lambda repressor and cro-components of an efficient molecular switch. Nature 294: 217-223.

Keller, E.B. and W.A. N oon. 1984. Intron splicing: A conserved internal signal in introns of animal pre-mRNAs. Proc. Natl. Acad. Sci. 81: 7417.

Kohtz, J.D., S.F. Jamison, C.L. Will, P. Zuo, R. Lührmann, M .A. Garcia-Blanco, and J.L. M anley. 1994. Protein-protein interactions and 5 '-splice-site recognition in mammalian mRN A precursors. Nature 368: 119-124.

Kramer, A. 1992. Purification of splicing factor SF1, a heatstable protein that functions in the assembly of a presplicing complex. Mol. Cell. Biol. 12: 4545-4552.

- - - 1996. The structure and function of proteins involved in mammalian pre-mRNA splicing. Annu. Rev. Biochem. 65: 367-409.

Lee, C.-G., P.D. Zamore, M.R. Green, and J. Hurwitz. 1993. RNA annealing activity is intrinsically associated with U2AF. J. Biol. Chem. 268: 13472-13478.

Madhani, H.D. and C. Guthrie. 1994. Dynamic RNA-RNA interactions in the spliceosome. Annu. Rev. Genet. 28: 1-26.

Michaud, S. and R. Reed. 1991. An ATP-independent complex commits pre-mRN A to the mammalian spliceosome assembly pathway. Genes \& Dev. 5: 2534-2546.

M oore, M.J., C.C. Query, and P.A. Sharp. 1993. Splicing of precursors to mRNAs by the spliceosome. In The RNA world (ed. R.F. Gesteland and J.F. Atkins), pp. 303-357. Cold Spring Harbor Laboratory Press, Cold Spring Harbor, NY.

M ount, S.M ., I. Pettersson, M. Hinterberger, A. Karmas, and J.A. Steitz. 1983. The U1 small nuclear RNA-protein complex selectively binds a 5' splice site in vitro. Cell 33: 509-518.
Patterson, B. and C. Guthrie. 1991. A U-rich tract enhances usage of an alternative $3^{\prime}$ splice site in yeast. Cell 64: 181187.

Ptashne, M. 1984. Repressors. Trends Biochem. Sci. 142-145.

- - . 1992. A genetic switch, 2nd ed. Cell Press, Cambridge, MA.

Query, C.C., M.J. Moore, and P.A. Sharp. 1994. Branch nucleophile selection in premRNA splicing: Evidence for the bulged duplex model. Genes \& Dev. 8: 587-597.

Query, C.C., S.A. Strobel, and P.A. Sharp. 1996. Three recognition events at the branch-site adenine. EMBO J. 15: 13921402.

Reed, R. 1989. The organization of $3^{\prime}$ splice-site sequences in mammalian introns. Genes \& Dev. 3: 2113-2123.

-_- 1996. Initial splice-site recognition and pairing during pre-mRNA splicing. Curr. Opin. Genet. Dev. 6: 215-220.

Reed, R. and T. Maniatis. 1985. Intron sequences involved in lariat formation during pre-mRN A splicing. Cell 41: 95-105.

-_- 1988. The role of mammalian branchpoint sequences in pre-mRN A splicing. Genes \& Dev. 2: 1268-1276.

Ruskin, B. and M.R. Green. 1985. Role of the $3^{\prime}$ splice site consensus sequence in mammalian pre-mRNA splicing. Nature 317: 732-734.

Rymond, B.C. and M. Rosbash. 1992. Y east pre-mRN A splicing. In The molecular and cellular biology of the yeast Saccharomyces: Gene expression (ed. E.W. Jones, J.R. Pringle, and J.R. Broach), pp. 143-192. Cold Spring Harbor Laboratory Press, Cold Spring Harbor, NY.

Séraphin, B., L. Kretzner, and M. Rosbash. 1988. A U 1 snRN A: Pre-mRN A base pairing interaction is required early in yeast spliceosome assembly but does not uniquely define the $5^{\prime}$ cleavage site. EMBO J. 7: 2533-2538.

Siliciano, P.G. and C. Guthrie. 1988. 5' splice site selection in yeast: Genetic al terations in base-pai ring with $U 1$ reveal additional requirements. Genes \& Dev. 2: 1258-1267.

Singh, R., J. Valcarcel, and M.R. Green. 1995. Distinct binding specificities and functions of higher eukaryotic polypyrimidine-tract binding proteins. Science 268: 1173-1176.

Smith, C.W.J. and B.N. N adal-Ginard. 1989. Mutualy exclusive splicing of $\alpha$-tropomyosin exons enforced by an unusual lariat branch point location: Implications for constitutive splicing. Cell 56: 749-758.

Staknis, D. and R. Reed. 1994. SR proteins promote the first specific recognition of pre-mRNA and are present together with $U 1$ snRN $P$ in a general splicing enhancer complex. Mol. Cell. Biol. 14: 7670-7682.

Val cárcel, J., R.K. Gaur, R. Singh, and M.R. Green. 1996. Interaction of $U 2 A F^{65} R S$ region with pre-mRNA of branch point and promotion base pairing with U2 SnRNA. Science 273: 1706-1709.

Wu, J.Y. and T. Maniatis. 1993. Specific interactions between proteins implicated in splice site selection and regulated alternative splicing. Cell 75: 1061-1070.

Zamore, P.D. and M.R. Green. 1989. Identification, purification, and biochemical characterization of U 2 small nuclear ribonucleoprotein auxiliary factor. Proc. Natl. Acad. Sci. 86: 9243-9247.

Zamore, P.D., J.G. Patton, and M.R. Green. 1992. Cloning and domain structure of the mammalian splicing factor U2AF. Nature 355: 609-614.

Zhuang, Y. and A.M. Weiner. 1986. A compensatory base change in $U 1$ snRNA suppresses a 5 ' splice site mutation. Cell 46: 827-835.

Zhuang, Y., M. Goldstein, and A.M. Weiner. 1989. UACUAAC is the preferred branch site for mammalian mRNA splicing. Proc. Natl. Acad. Sci. 86: 2752-2756. 


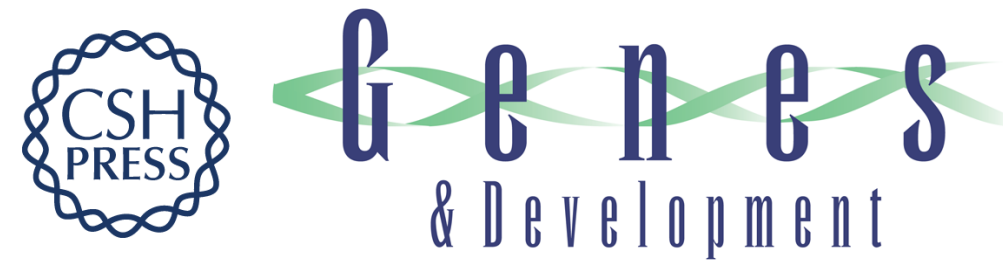

\section{A cooperative interaction between U2AF65 and mBBP/SF1 facilitates branchpoint region recognition}

J. Andrew Berglund, Nadja Abovich and Michael Rosbash

Genes Dev. 1998, 12:

References This article cites 42 articles, 21 of which can be accessed free at:

http://genesdev.cshlp.org/content/12/6/858.full.html\#ref-list-1

License

Email Alerting Receive free email alerts when new articles cite this article - sign up in the box at the top Service right corner of the article or click here.

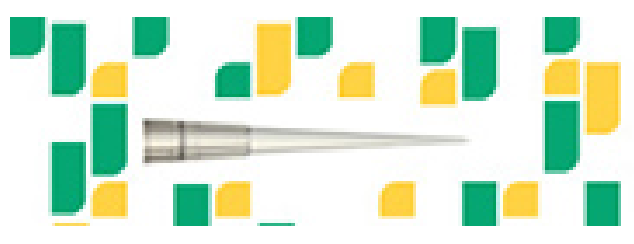

Focused on your science. 